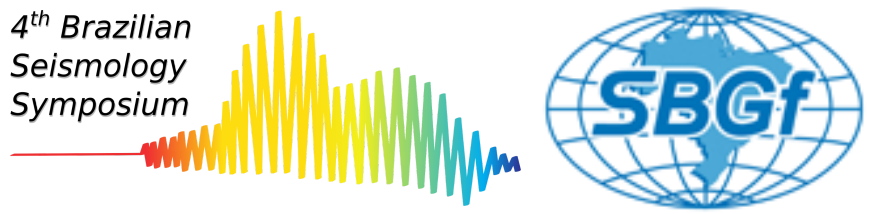

\title{
Analysis of the influence of the mantle shear-wave velocity structure on travel time and amplitude of SS-waves and its precursors: implications for the determination of the
} mantle transition zone

\author{
Klaus Contiero', Carlos A. M. Chaves ${ }^{1},{ }^{1}$ Universidade de São Paulo \\ Copyright 2021, SBGf - Sociedade Brasileira de Geofísica. \\ This paper was prepared for presentation during the $17^{\text {th }}$ International Congress of the Brazilian Geophysical Society held in Rio de Janeiro, Brazil, 8-11 November 2021 (Online Event). \\ Contents of this paper were reviewed by the Technical Committee of the $17^{\text {th }}$ International Congress of the Brazilian Geophysical Society and do not necessarily represent any position of \\ the SBGf, its officers or members. Electronic reproduction or storage of any part of this paper for commercial purposes without the written consent of the Brazilian Geophysical Society is \\ prohibited.
}

The mantle transition zone (MTZ) is characterized by phase changes in olivine, the most abundant mineral in the mantle, and plays a key role in determining the mantle convection style, either layered convection or whole-mantle convection. Understanding the mantle transition zone structure can provide important constraints on the convection style that prevails in the mantle, a fundamental open problem in geodynamics. In global studies, the MTZ is primarily estimated from the analysis of the so-called SS precursors, which are small-amplitude signals due to the underside shear- wave reflections off the 410 and $660 \mathrm{~km}$ discontinuities (i.e., S410S and S660S), midway between earthquakes and seismic stations. SS precursors traveltimes are measured using a sum (i.e., stack) of thousands of waveforms in which coherent small-amplitude signals are raised above noise level. While stacking is a simple procedure in principle, rigorous analyses of uncertainties and the presence of artifacts have not received much attention. Moreover, the translation of the traveltimes of the upper mantle reflections into estimates of the depths of discontinuities relies on corrections for the contribution of wave speed heterogeneity in the crust and mantle. Usually, tomographic models (with severe resolution issues of their own) are used and ray-theoretical traveltime calculations are inaccurate due to the broad Fresnel zones of SS phases. In this study, to expose the inaccuracies of waveform stacking and ray-theoretical limitations to estimating the influence of shear-wave velocity heterogeneity on travel times, we carry out spectral-element method (SEM) waveform simulations. First, we explore the influence of narrow structures of different sizes and velocity perturbations in the mantle (e.g., plumes and slabs) on the travel time and amplitude of SS and its precursors, by placing 110 virtual stations at intervals of $1^{\circ}$ on Earth's surface along the equator. Our travel time perturbations and amplitude ratios estimates of the SS and its precursors show important finite-frequency, focusing and defocusing effects, which may complicate the waveform stacking procedure. Then, for six different Earth shear-wave velocity models (PREM, S40RTS, TX2015, SEMUCB, GLOBE-rani, S362ANI+M), we compute transverse-component waveforms for 50 shallow earthquakes uniformly distributed over the globe, which were recorded by 16,200 virtual seismic stations on a global grid with $2^{\circ} \times 2^{\circ}$ spacing. Our preliminary analysis shows that inaccuracies in the ray-theoretical corrections do not severely influence the resolution of long-wavelength MTZ maps, yet shortest wavelengths are affected by the velocity correction on travel times. 